\title{
Tipping points in Complex Coupled Life-Environment Systems
}

\author{
Iain S. Weaver* and James G. Dyke \\ School of Electronics and Computer Science, University of Southampton, UK, SO17 1BJ \\ *isw1g10@soton.ac.uk
}

\begin{abstract}
Simple models of complex phenomena provide powerful insights and suggest low-level mechanistic descriptions. The Earth system arises from the interaction of subsystems with multi-scale temporal and spatial variability; from the microbial to continental scales, operating over the course of days to geological time. System-level homeostasis has been demonstrated in a number of conceptual, artificial life, models which share the advantage of a thorough and transparent analysis. We reintroduce a general model for a coupled life-environment model, concentrating on a minimal set of assumptions, and explore the consequences of interaction between simple life elements and their shared, multidimensional environment. In particular stability, criticality and transitions are of great relevance to understanding the history, and future of the Earth system. The model is shown to share salient features with other abstract systems such as Ashby's Homeostat and Watson and Lovelock's Daisyworld. Our generic description is free to explore high-dimensional, complex environments, and in doing so we show that even a small increase in the environmental complexity gives rise to very complex attractor landscapes which require a much richer conception of critical transitions and hysteresis.
\end{abstract}

\section{Introduction}

The principle that environmental factors affect life is evident throughout the biosphere both regionally and globally, and throughout Earth's history (Gaston 2000). Variables such as temperature and soil or atmospheric composition determine whether an organism can proliferate, and populations will develop to some resource limited level. Climatic shifts between, for example, greenhouse and icehouse states are accompanied by mass extinction (Haywood 2004). Indeed this principle is at the core of environmental niche modelling, where the distribution of species in the space of significant environmental factors, their realized-niche, is used to predict the spatial species distribution (Thomas et al. 2004). The influence of life on its environment however, and whether it is expected to have a stabilising, or destabilising effect is less clear. Lovelock and Margulis's (1974) original "Gaia Hypothesis" focused on the extent to which the emergence of life has promoted a self-regulating, or homeostatic system. In this work we consider the ability of a coupled life- environment system to generate stabilising feedback loops in increasingly complex environments under a minimal set of assumptions.

The Gaia-hypothesis proposes that life may self-organise into complex, self-regulating systems with maintain the habitability of their environment and at the higher level contribute to regulation of variables globally conducive for life to flourish. Controversy followed as it is unclear how regulatory mechanisms might emerge without the need for system-wide cooperation which would contradict the principle of natural selection at the species level (Doolittle 1981). Watson and Lovelock's (1983) "Daisyworld" took the first step towards addressing this with an abstract coupled lifeenvironment model whose biota consists of two species of daisy which exert a unidirectional force on their shared environment, reduced to a single temperate variable. Black daisies absorb a large amount of energy, and have a warming effect compared to white daisies which have a high albedo and an overall cooling effect. If the species are organised such that black daisies out-compete white daisies at low temperatures, the model shows that infinitesimally differentiated species can establish reign-control over their environment (Harvey 2004); a small reduction in global temperature allows the black absorptive daisies to proliferate and visaversa for increases in temperature. In this way, each species holds a reign, opposing changes in one direction such that the planetary temperature is not just stable, but robust to a range of external perturbations. There have been a number of extensions and developments of the original Daisyworld model (see Wood et al. (2008) for a review), some of which has been undertaken within the field of artificial life. Dyke et al. (2007) for example allow external perturbation to vary on timescales comparable to changes in the biota, while Williams and Noble (2005) extend the model to enable stochastic evolution of daisy species.

We aim to address two short-comings of this model in detail. Firstly, while useful in its transparency, the behaviour of such a one-dimensional dynamical system is extremely limited. Iconic phenomena such as hysteresis loops are a common metaphor for transitions in very complex systems, 
although do not take into account the additional degrees of freedom of high dimensional systems which may exhibit cyclical, complex or chaotic behaviour, inaccessible to such a simple metaphor. It is therefore unclear the extent to which this picture fits such high-dimensional systems. On the other hand, while more complicated many-body systems benefit from a much richer zoo of emergent behaviours, it typically comes at the expense of transparency, exchanging generality for a more faithful representation of a specific system. Along with increasingly complex environments, the relevance of the reign-control mechanism to very much larger populations of diverse biotic elements in unclear. Daisyworld does not address the mechanism by which the pair of antagonistic species might emerge. While later work makes clear that the mechanism is not unique to a two-species model, a persistent theme of such models is that they are in some sense designed (Lenton 1998).

Along with these key points, we explore the nature of critical transitions or "regime shifts" (Williams and Lenton 2010). We utilize a Daisyworld-type model first introduced in Dyke (2010) then extended in Dyke and Weaver (2013) in order to assess to what extent iconic phenomena such as hysteresis loops, which are a common metaphor for transitions in very complex systems, are appropriate when considering higher dimensional systems. We will show that even a small increase in the environmental complexity generates surprisingly detailed, and complex attractor landscapes. Understanding the way in which we traverse the space of environmental variables calls for a richer conception of critical transitions and hysteresis. In short we find that some critical transitions have little impact on system behaviour while others can produce large changes from states which may not be recoverable, resulting in asymmetrical transitions. Importantly, there appears no immediate way in which to differentiate between them and thus our results may be interpreted as urging caution for the use of early warning signals for complex systems.

\section{Model formulation}

Dyke's (2010) "Daisystat" model in single environmental variable mode can be understood as a simplified Daisyworld model, very similar to the Artificial Life model of McDonald-Gibson et al. (2008). A population of simple organisms is affected by their environment such that they thrive in the vicinity of their ecological niche, and in turn influence the environment through simple, linear feedbacks. The key differences between the Daisystat model, and Daisyworld are that Dyke's (2010) enables the description of higher dimensional environments and rather than prescriptive feedbacks, the model employs random lifeenvironment interactions.

Throughout this work, we describe the distribution of biota through $K$ variables, $\boldsymbol{\alpha}$, denoting the overall activity of individual elements, or populations, influenced by the state

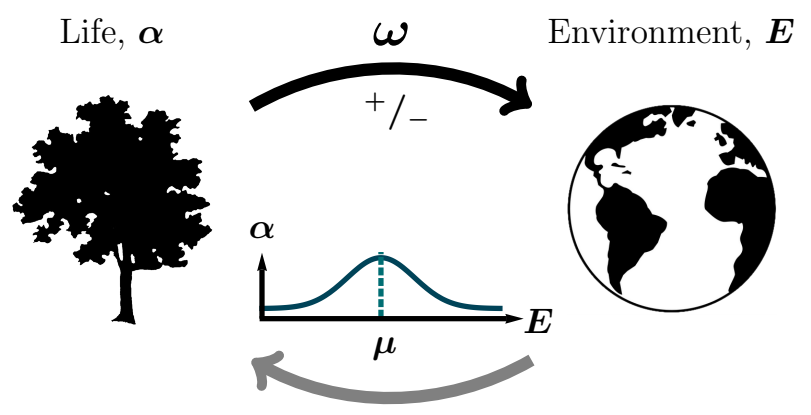

Figure 1: In the simplest case, biotic elements, $\boldsymbol{\alpha}$, have an increasing or decreasing, $\boldsymbol{\omega}$, effect on their environment $\boldsymbol{E}$. In turn, they are only abundant over a finite range of the environmental variables, centred on their fundamental niche, $\mu$.

of their shared environment, represented the $N$ variables in the vector $\boldsymbol{E}$, where we have used boldface notation to denote vectors, and will reference individual elements with subscripted indices.

$$
\boldsymbol{X}=\left[\begin{array}{c}
X_{1} \\
X_{2} \\
\vdots \\
X_{n} .
\end{array}\right]
$$

The first principle assumption is that components of the biota are only significantly abundant, or active, in the vicinity of their ideal environmental conditions, or niche. The second assumption is that the environment is itself influenced by the biota; environmental variables may be decreased or increased by the individual biotic elements, through consumption, excretion or some other process with no bias towards positive or negative feedback. In essence, the model consists of these two principle assumptions, illustrated by Fig. 1. This section elaborates on these assumptions, along with their consequential behaviour.

i) Environment affects life Each element of the biota only has a significant presence in a narrow range of environmental conditions wherein it may proliferate, respire, or is otherwise active. As environmental conditions depart this niche, the activity is reduced until the population recedes or becomes dormant such that its presence is negligible. While many species may occupy a wide range of niches, we may add an additional constraint that there is a limited range of environmental conditions which are conducive to life at all, such as the conditions to maintain liquid water. This confines the niche for any population to some volume of the space of environmental variables within which life may exist, known as the essential range (Ashby 1952), given by the range $[0: R]$ for simplicity.

A simple choice of function to describe the changes in the 


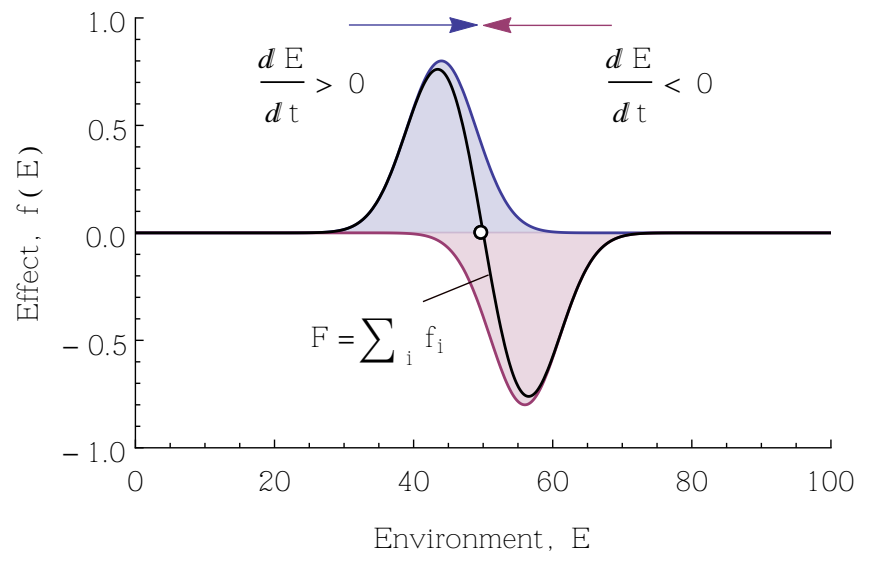

(a) Two-species with antagonistic effects.

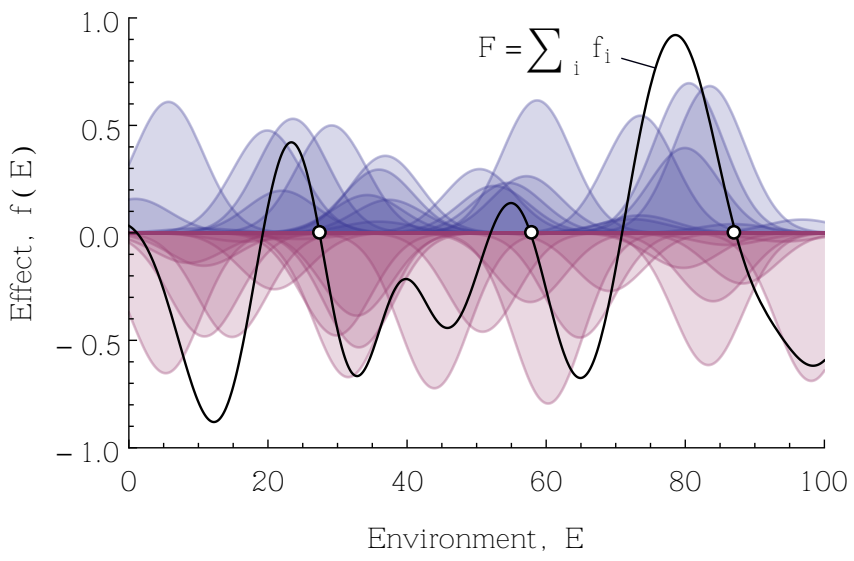

(b) 50-species with random effects.

Figure 2: Activity of individuals with increasing (blue) and decreasing (red) effects on their environment, along with the net effect $F(E)=\sum_{i} f_{i}(E)$. Fixed points, shown by open circles, occur where the sum of individual effects is exactly zero. Fig. 2a is analogous to Daisyworld and illustrates the reign-control mechanism, along with a single fixed point. Fig. $2 b$ shows large numbers of species with random effects (with no preference for negative, or positive feedback), demonstrating the emergence of such points by chance.

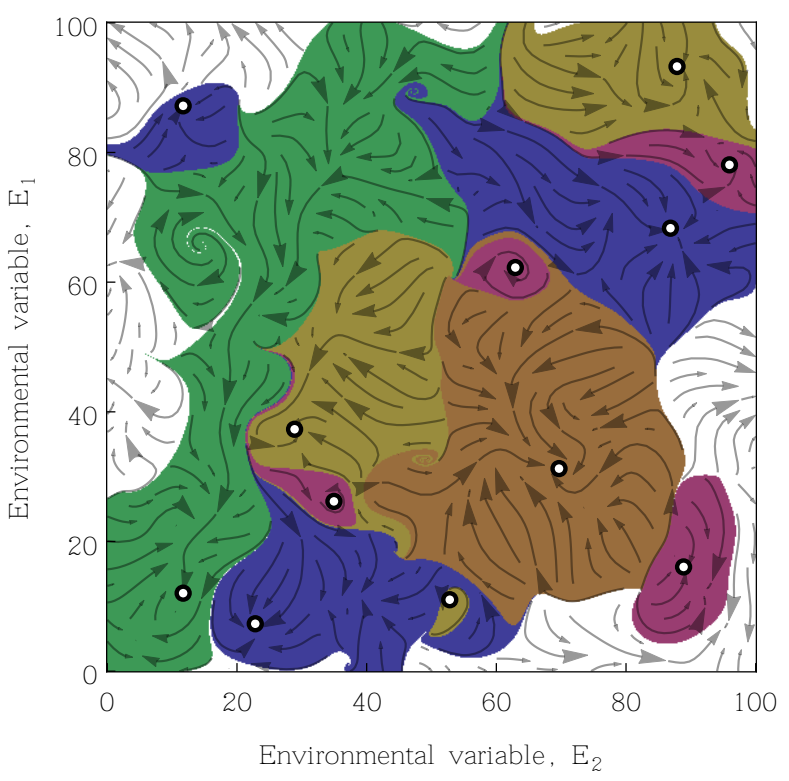

Figure 3: The two environmental variable model with no external perturbation, $\boldsymbol{P}=\mathbf{0}$. Points indicate the position of stable fixed points, where $\boldsymbol{F}(\boldsymbol{E})=\mathbf{0}$ and $\nabla \boldsymbol{F}<\mathbf{0}$. Shaded regions show the basin of attraction for each point indicating that a model initiated within the region will arrive at the fixed point. Increasingly positive or negative perturbations will influence the shape of the attractor space such that some attractors will disappear, and new attractors may even emerge, resulting in rapid transitions. biota would be a linear relaxation towards some steady-state;

$$
\tau_{\alpha} \frac{\mathrm{d} \alpha_{i}(t)}{\mathrm{d} t}=\alpha^{*}\left(\boldsymbol{E}, \boldsymbol{\mu}_{i}\right)-\alpha_{i}(t)
$$

where $\tau_{\alpha}$ defines the timescale of changes in the population $\alpha_{i}(t)$ towards its steady-state value, $\alpha^{*}(\boldsymbol{E}, \boldsymbol{\mu})$ is the steadystate distribution about the individual niche $\boldsymbol{\mu}_{i}$. It has been shown that the specific choice of this distribution is unimportant providing it has a well defined variance (Dyke and Weaver 2013). In this instance we choose a Gaussian distribution with characteristic width $\sigma_{E}$, centred on $\boldsymbol{\mu}$ chosen randomly in the interval $[0: R]$, the essential range.

$$
\alpha^{*}(\boldsymbol{E}, \boldsymbol{\mu})=\exp \left(-\frac{|\boldsymbol{E}-\boldsymbol{\mu}|^{2}}{2 \sigma_{E}^{2}}\right)
$$

ii) Life affects environment On the other hand, the biota has an effect on their shared environment. Individual populations may modify environmental variables independently, having either an increasing or decreasing effect. Black daisies in Watson and Lovelock's (1983) Daisyworld for example absorb a large amount of radiation compared to white daisies, having an increasing effect on temperature. In the simplest case, the biota has an effect, $f$, in proportion to their activity

$$
\boldsymbol{f}_{i}(t)=\boldsymbol{\omega}_{i} \alpha_{i}(t)
$$

where $\boldsymbol{\omega}_{i}$ is the effect of an individual on the environment $\boldsymbol{E}$, chosen randomly from the interval \pm 1 . The net effect of the biota on the environment, $\boldsymbol{F}$, is simply found by summing individual contributions

$$
\boldsymbol{F}(t)=\sum_{i=1}^{K} \boldsymbol{f}_{i}(t) .
$$



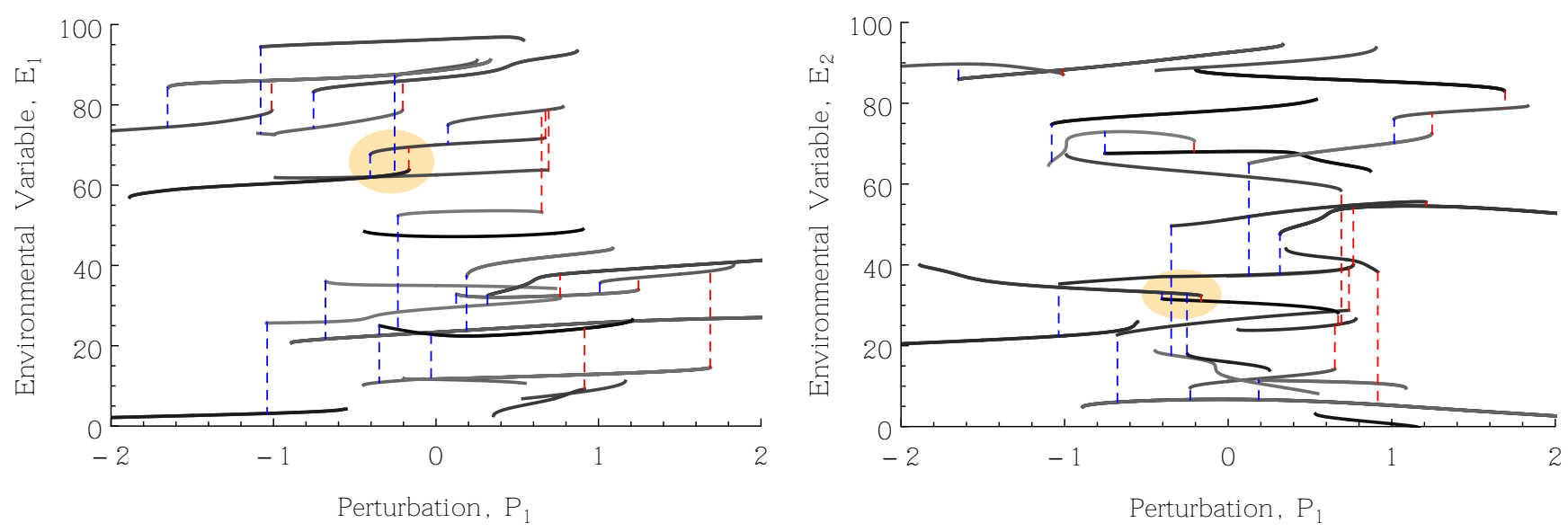

Figure 4: The states and transitions which exist in a two-dimensional environment model for a range of $P_{1}$, where $P_{2}=0$. States are shaded randomly to help differentiate them, and end abruptly where states become unstable and transition outside the essential range, as opposed to towards another stable state. Transitions to other states are colored red and blue where the transition is encountered by increasing and decreasing perturbation respectively. Importantly, not all transitions are symmetrical. Several transitions occur from states which may not be recovered by trivially applying an opposing perturbation as would be expected in the case of a one-dimensional system. Indeed some states undergo no transitions at all, besides those which would drive the system outside the essential range. The basins of attraction which lead to the hysteresis loop marked are represented by Fig. 5 .

Additionally, each of the environmental variables may be affected by some external abiotic perturbing force, $\boldsymbol{P}$. In the original Daisyworld model, the single environmental variable was temperature, and the perturbing force was insolation, the influence of Daisyworld's star. Other perturbing effects may include flux of chemicals or gasses, such as by volcanic or anthropogenic emission. The net change in the environment is the sum of these features

$$
\tau_{E} \frac{\mathrm{d} \boldsymbol{E}(t)}{\mathrm{d} t}=\boldsymbol{P}+\boldsymbol{F}(t)
$$

where $\tau_{E}$ is the characteristic timescale for changes in environmental variable $i$, chosen to be equal between variables for convenience, and $F_{i}$ is the sum of effects from the biota.

It is important to establish the relationship between the times scales of changes in the biota, $\tau_{\alpha}$, and their shared environment, $\tau_{E}$. Progress towards establishing the behaviour of such models has been demonstrably simpler by assuming a separation of timescales $\tau_{E} \gg \tau_{\alpha}$. In the Daisyworld model for example, this leads directly to an analytical solution (Weaver and Dyke 2012). In this limit, the populations $\alpha_{i}(t)$ quickly adjust to their steady-state values $\alpha^{*}\left(\boldsymbol{E}, \boldsymbol{\mu}_{i}\right)$, removing the time dependence in $\boldsymbol{F}(t) \rightarrow \boldsymbol{F}(\boldsymbol{E})$. Furthermore, the summed effect $\boldsymbol{F}$ is normalised to have a variance $\sigma_{F}^{2}=1$ for convenience.

Similarities with Watson and Lovelock's (1983) Daisyworld are clear for simple, one-dimensional environments. Fig. 2 illustrates the individual effects of the individual populations, $f_{i}$, along with the total effect, $F$. Fig. 2a shows the Daisyworld control mechanism, so called reign-control.
The two populations exert opposing forces on their environment, where the population with a positive effect is abundant at low ranges, while the negative effect dominates for larger values. This system naturally finds stability at a point where these effects are exactly in balance, somewhere between the niches of the opposing populations. Fig. $2 \mathrm{~b}$ shows $50 \mathrm{ran}$ dom populations (random in both their niche, and influence of the environment). Importantly, fixed points occur where the net effect, $F$, crosses zero, and are furthermore stable only when the gradient is negative. In the case of Daisyworld, it may be remarked that the single fixed point is prescribed in the model formulation. However, previous work has concentrated on the emergence of these points from interactions between many populations and their shared environment by chance, finding them to be a generic property of such models, largely independent of the dimensionality of $\boldsymbol{E}$ (Dyke and Weaver 2013).

\section{Higher Dimensions}

Typically, illustrations of hysteresis involve a single variable. Such examples however bely the much more colourful behaviour which emerges in higher dimensional systems. Firstly, it has been shown that the expected number of fixed points increases exponentially with the dimensionality of the environment (Dyke and Weaver 2013), resulting in very many more stable states. This may be contrary to intuition as a fixed point must be stationary in all dimensions simultaneously, and appears exponentially unlikely with more complex environments. However, this is opposed by the increase 

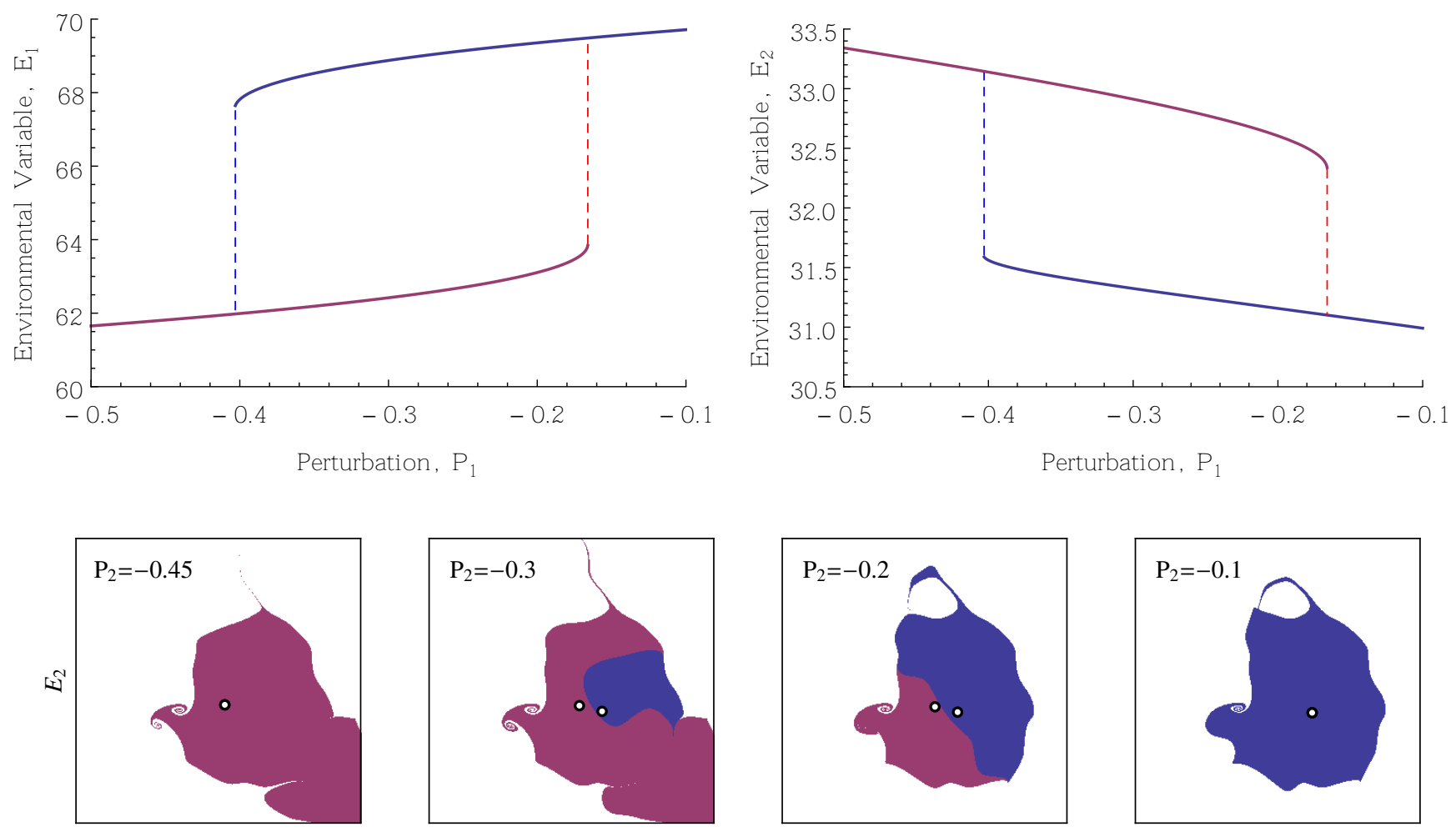

Figure 5: Colored regions correspond to the basins of attraction of the two fixed points (shown by open circles) highlighted in Fig. 4. As the perturbation is varied, one fixed point vanishes, and its basin of attraction is encompassed by that of the other fixed point, resulting in a transition. In this instance, the transition is roughly symmetrical and the previous state may be recovered by reversing the perturbation.

in volume of the environment $\boldsymbol{E}$, which enables many more configurations of the biota. Here, we demonstrate the complicated network of transitions between these states with increasing or decreasing perturbations. Fig. 3 shows a randomly initialised two-dimensional system of fixed points for the model subjected to no external perturbation. The response of these fixed points to perturbation is demonstrated by Fig. 4, showing the same two-dimensional system for increasing and decreasing perturbations in one direction. The complicated behaviour is perhaps best interpreted in terms of attractors, where transitions occur when a basin of attraction is fully succeeded by another, illustrated by Fig. 5 for a roughly symmetrical transition between two states. Some notable features of Fig. 4 include

Density of states The stable states consistent with a particular driving force exhibits great diversity. Rather than bistability, Fig. 4 shows over a dozen stable states at a given perturbation. Furthermore Dyke and Weaver (2013) finds the density of stable states increases exponentially with environmental complexity. It is not unreasonable to expect higher dimensional environments to house hundreds of vi- able stable states. Another interesting observation of Fig. 4 is that the density of stable states diminishes (exponentially) with increasing perturbation strength. In a one dimensional system for example, the mean number of stable states in a unit interval is given by

$$
n=\frac{1}{2 \sqrt{2} \pi \sigma} \exp \left(-\frac{P^{2}}{2}\right)
$$

while is illustrated in Fig. 6. It is intuitive that the summed contributions of random, uncorrelated effects is Gaussian distributed, and therefore the density of points with a strong enough effect to oppose increasing perturbations decreases.

Step size The geological record is punctuated by abrupt and in some sense catastrophic transitions (Alley et al. 2003). Such events correspond to large, quantitative changes although in a high dimensional space it is important to note that such steps may not necessarily be large in all dimensions. Fig. 4 illustrates that many transitions in which one or the other environmental variable undergoes a very small change. This observation highlights the importance in identifying the significant axes of change in understanding high 

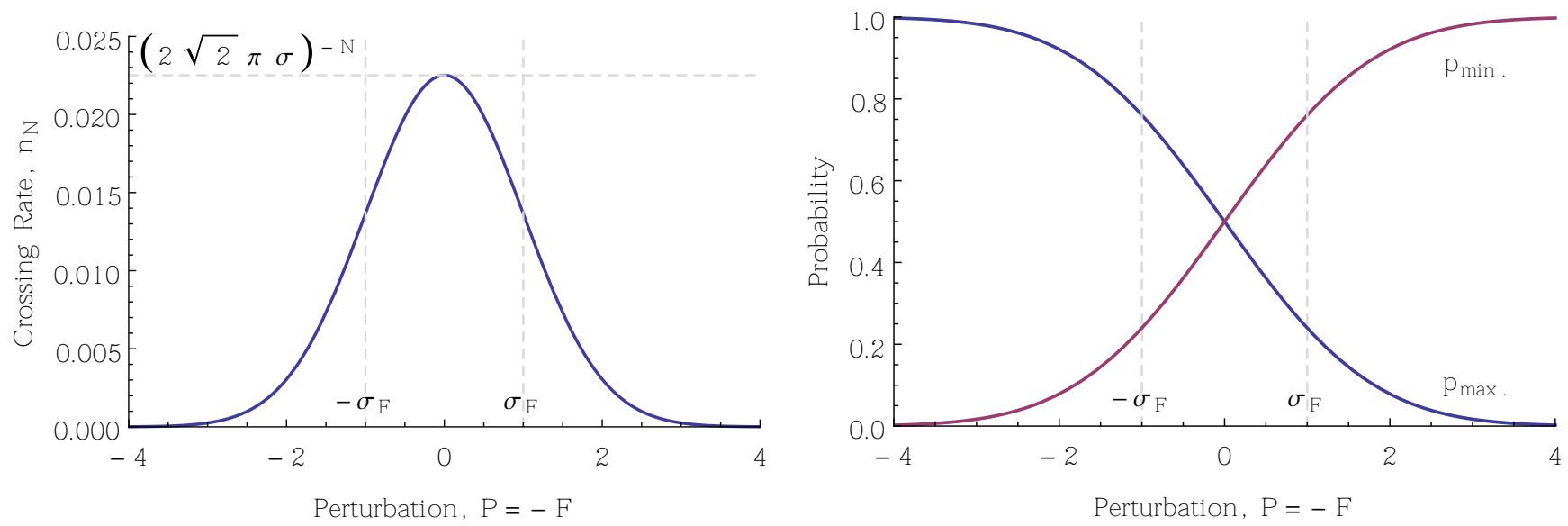

Figure 6: Eq. (6) (left) shows the density of fixed points over a range of perturbations to be Gaussian distributed, such that exponentially fewer stable states are available in the face of large perturbations. Additionally we can derive the probability of a transition occurring in the increasing or decreasing $P$-direction as a function of $P$ (right). While extrema are equally likely at any value of $P$, they are more likely to be maxima where $P$ is negative, and minima where it is positive. Transitions encountered by increasing $P$ are therefore more likely when $P$ is positive and visa-versa. The width of the functions is related to the variance of $F$, which is normalised to $\sigma_{F}^{2}=1$.

dimensional transitions as opposed to examining a lower dimensional projection. It is also interesting to examine the probability that a transition occurs in the direction of increasing perturbation (local minima of $\boldsymbol{F}(\boldsymbol{E})$ ) or in the decreasing direction (local maxima). To achieve this, we examine the correlations between $F$ and it's second derivative, $F^{\prime \prime}$ by formulating the covariance matrix, the result of which is shown in Fig. 6. This illustrates that positive forcing is more likely to encounter a local minimum, and therefore a transition caused by increasing $P$ while the reverse is true for negative forcing.

Reversibility The one-dimensional hysteresis metaphor describes a symmetrical loop, emphasising only that systems may be bistable over a range of external perturbation, and that sufficiently strong driving can move the system between states. However, while Fig. 5 shows such transitions exist, the two dimensional picture significantly obfuscates this as along with simple hysteresis loops, we see large numbers irreversible regime shifts. This occurs where driving in a particular environmental variable will not revert the system to its original state. This result would have particularly important consequences to real-world transitions. Transitions in lakes to eutrophic states for example reduce biodiversity and cause difficulty in water treatment (Wang et al. 2012). Reversing these transitions is a key concern, and this model emphasises the importance of a thorough understanding of the system in question. A system collapsed by strong driving in one dimension may not be recoverable simply by reversing the effect.

\section{Discussion}

We have shown that self-regulation is a mechanism which may arise from a large population of random life elements, and explored this mechanism in a multi-dimensional environment. In particular, we note three important differences between the one-dimensional picture of hysteresis between bistable states; the magnitude of a regime-shift following a transition is highly variable, returning to a previous state after a transition may be impossible, and the density of viable states for a given perturbation is exponentially large with the number of environmental variables. These points prompt a number of questions when considering transitions in climatic or ecosystems; which aspects of the environment if any are expected to undergo catastrophic changes? Can the previous state be recovered, and if so, which are the important dimensions of control? How many alternative states are consistent with external forces, and therefore stable?

Two main questions are left open in our implementation. While it has been shown that the choice of function for the steady state populations about their niche is unimportant, a factor which would convolute this such as interspecies competition are not resolved. In this sense, we do not distinguish between the fundamental niche, the environmental conditions in which the species can survive and proliferate, and realized niche which is influenced by external factors such as interspecies competition and predation. While we mediate all biotic interactions through the environment, previous studies have concentrated on the individual species interactions, such as McDonald-Gibson et al. (2008) and Dyke et al. (2007) where increased interspecies competition appears to accentuate the homeostatic properties of a reign-control sys- 


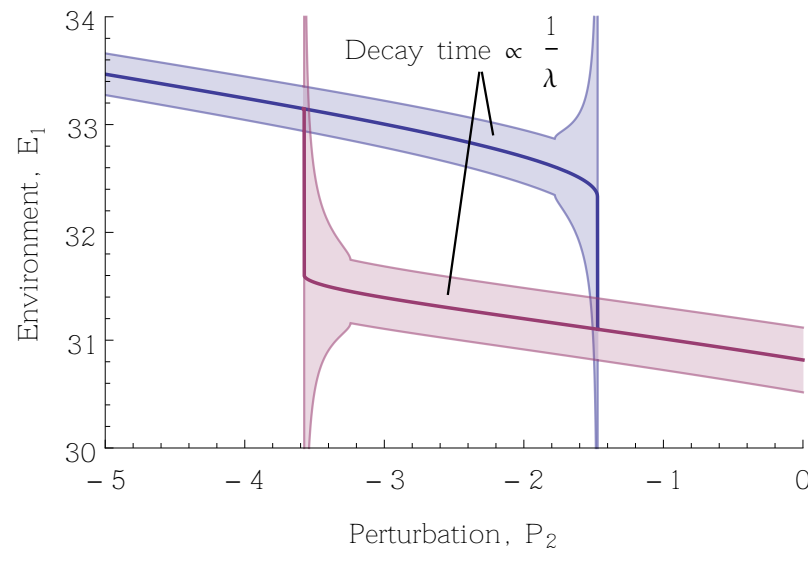

Figure 7: Shaded regions indicate the time taken for the system to recover from small perturbations from the fixed points of Fig. 5. The decay time is inversely proportional to the real part of the largest eigenvalue of the Jacobian evaluated at the fixed point. The discontinuity is caused by the transition between an over-damped system, where eigenvalues are real and perturbations decay exponentially, and an under-damped system where complex eigenvalues result in damped oscillations.

tem, despite other approaches which argue that increasingly connected systems lose stability (May 1972).

Also, the extent to which the separation of time scales between changes in the environment and biota are important to the model dynamics is unclear. While it has no influence on the model fixed points, illustrations of basins of attraction, such as Fig. 5 are expected to change significantly, and there is no clear way to represent them in a $N+K$ dimensional system. However, some progress may be possible by assuming the simplest case of linear relaxation of populations towards some steady state value. Starting from Eq. (1), it can be shown that such a case removes the need to resolve the individual populations $\boldsymbol{\alpha}$, reducing to a $2 N$ dimensional system. Multiplying by $\boldsymbol{\omega}_{i}$ and summing over the population gives

$$
\begin{aligned}
\tau_{\alpha} \sum_{i} \boldsymbol{\omega}_{i} \frac{\mathrm{d} \alpha_{i}(t)}{\mathrm{d} t} & =\sum_{i} \boldsymbol{\omega}_{i} \alpha^{*}\left(\boldsymbol{E}, \boldsymbol{\mu}_{i}\right)-\sum_{i} \boldsymbol{\omega}_{i} \alpha_{i}(t) \\
\tau_{\alpha} \frac{\mathrm{d} \boldsymbol{F}(t)}{\mathrm{d} t} & =\boldsymbol{F}^{*}(\boldsymbol{E})-\boldsymbol{F}(t)
\end{aligned}
$$

by the definition for $\boldsymbol{F}$ of Eq. (4). Previous work has shown that the relationship between the time scales of changes in the biota and changes in external perturbation set limits on the ability of a system to self-regulate, and invite a range of new phenomena to emerge (Weaver and Dyke 2012).

Critical slowing-down (CSD) (Lenton 2011) refers to the long relaxation time of near-critical systems, those which are approaching climatic or ecosystem transitions. As natural and anthropogenic pressures stress aspects of the Earth system, it has been shown that certain time series find an increase in the auto-correlation coefficient precedes such transitions (Dakos et al. (2008) analyze eight ancient abrupt climate shifts). This signal suggests that the relaxation of the system towards its steady state slows as it approaches a regime shift. Fig. 7 illustrates the decay time of the system shown in Fig. 5 and verifies that the type of system described in this work exhibits this signal. The eigenvalues of the Jacobian in the vicinity of a fixed point yield information not only pertaining to the stability of the point, but also provide estimates of the decay time of small fluctuations from the fixed point. A large decay time indicates the system is in some sense slow. However it appears to posses no further information pertaining to the direction, magnitude or reversibility of the transition; clearly important and relevant questions when considering transitions in real systems.

\section{Acknowledgements}

This work was supported by an EPSRC Doctoral Training Centre grant (EP/G03690X/1).

\section{References}

Alley, R. B., Marotzke, J., Nordhaus, W., Overpeck, J., Peteet, D., Pielke, R., Pierrehumbert, R., Rhines, P., Stocker, T., Talley, L., et al. (2003). Abrupt climate change. science, 299(5615):2005-2010.

Ashby, W. (1952). Design for a brain. Wiley.

Dakos, V., Scheffer, M., van Nes, E. H., Brovkin, V., Petoukhov, V., and Held, H. (2008). Slowing down as an early warning signal for abrupt climate change. Proceedings of the National Academy of Sciences, 105(38):14308-14312.

Doolittle, W. F. (1981). Is nature really motherly. CoEvolution Quarterly, 29:58-63.

Dyke, J., McDonald-Gibson, J., Di Paolo, E., and Harvey, I. (2007). Increasing complexity can increase stability in a selfregulating ecosystem. In Advances in Artificial Life, pages 133-142. Springer.

Dyke, J. G. (2010). The daisystat: A model to explore multidimensional homeostasis. In Artificial Life XI, Proceedings of the Eleventh International Conference on the Simulation and Synthesis of Living Systems, pages 349-359. MIT Press, Cambridge MA.

Dyke, J. G. and Weaver, I. S. (2013). The emergence of environmental homeostasis in complex ecosystems. PLoS computational biology, 9(5):e1003050.

Gaston, K. J. (2000). Global patterns in biodiversity. Nature, 405(6783):220-227.

Harvey, I. (2004). Homeostasis and rein control: From daisyworld to active perception. In Proceedings of the Ninth International Conference on the Simulation and Synthesis of Living Systems, ALIFE, volume 9, pages 309-314.

Haywood, A. M. (2004). From greenhouse to icehouse: The marine eocene-oligocene transition. Antarctic Science, 16(4):585-586. 
Lenton, T. M. (1998). Gaia and natural selection. Nature, 394(6692):439-447.

Lenton, T. M. (2011). Early warning of climate tipping points. Nature Climate Change, 1(4):201-209.

Lovelock, J. E. and Margulis, L. (1974). Atmospheric homeostasis by and for the biosphere. Tellus Series B-Chemical and Physical Meteorology, 26(4):299-327.

May, R. M. (1972). Will a large complex system be stable? Nature, 238:413-414.

McDonald-Gibson, J., Dyke, J., Di Paolo, E., and Harvey, I. (2008). Environmental regulation can arise under minimal assumptions. Journal of theoretical biology, 251(4):653-666.

Thomas, C. D., Cameron, A., Green, R. E., Bakkenes, M., Beaumont, L. J., Collingham, Y. C., Erasmus, B. F., De Siqueira, M. F., Grainger, A., Hannah, L., et al. (2004). Extinction risk from climate change. Nature, 427(6970):145-148.

Wang, R., Dearing, J. A., Langdon, P. G., Zhang, E., Yang, X., Dakos, V., and Scheffer, M. (2012). Flickering gives early warning signals of a critical transition to a eutrophic lake state. Nature, 492(7429):419-422.

Watson, A. and Lovelock, J. (1983). Biological homeostasis of the global environment: the parable of daisyworld. Tellus $B$, 35(4):284-289.

Weaver, I. S. and Dyke, J. G. (2012). The importance of timescales for the emergence of environmental self-regulation. Journal of Theoretical Biology, 313(0):172 - 180.

Williams, H. and Noble, J. (2005). Evolution and the regulation of environmental variables. In Advances in Artificial Life, volume 3630, pages 332-341. Springer Berlin Heidelberg.

Williams, H. T. and Lenton, T. M. (2010). Evolutionary regime shifts in simulated ecosystems. Oikos, 119(12):1887-1899.

Wood, A. J., Ackland, G. J., Dyke, J. G., Williams, H. T. P., and Lenton, T. M. (2008). Daisyworld: a review. Reviews of Geophysics, 46:RG1001. 